\title{
THE UNIQUENESS QUESTION FOR WAVES AGAINST AN OVERHANGING CLIFF*
}

\author{
BY \\ R. SHERMAN LEHMAN
}

1. Introduction. For the problem of three-dimensional waves over sloping beaches Roseau [11] and Peters [10] have found explicit solutions for all slope angles. These solutions are in the form of integrals in the complex plane resembling those obtained in the inversion of the Laplace transform. It is not, however, clear that all solutions satisfying appropriate conditions can be represented in this way, and indeed in general the uniqueness question is still open. The purpose of the present paper is to study the uniqueness question in a particular case.

The problem of surface waves over sloping beaches when treated by the linearized theory leads to the question of determining a velocity potential $\Phi(x, y, z, t)$ which is a solution of Laplace's equation in the space variables $x, y$, and $z$ and satisfies two different boundary conditions on different parts of the boundary. Suppose the $z$-axis is taken along the shore, the $y$-axis is directed vertically upward with the free surface at $y=0$, and the $x$-axis is directed outward from the shore. Then on the free surface $y=0, x>0$

$$
\frac{\partial^{2} \Phi}{\partial t^{2}}+g \frac{\partial \Phi}{\partial y}=0
$$

where $g$ is the acceleration due to gravity. On the bottom the normal derivative $\partial \Phi / \partial n=0$. For a uniformly sloping beach with angle $\pi \alpha$ between the surface and bottom, the bottom is given by the equation $\theta=-\pi \alpha$, when $x=r \cos \theta, y=r \sin \theta, r>0$. If $\alpha=\frac{1}{2}$ the "bottom" becomes a vertical cliff, while for $\frac{1}{2}<\alpha<1$ the "bottom" becomes an overhanging cliff. Our considerations here will be primarily concerned with the case $\alpha=3 / 4$.

Restricting consideration to motion which is simple harmonic in the time $t$ and the space variable $z$, one seeks solutions of the form

$$
\Phi(x, y, z, t)=\varphi(x, y) \cos k z \cos (\sigma t+\beta) .
$$

Progressive waves having crests which at infinity make an arbitrary angle with the shore line can be constructed by taking linear combinations of standing waves of this type.

With an appropriate choice of units of length and time so that $\sigma^{2} / g=1$ one obtains the following mixed boundary value problem: Find the solutions of the equation

$$
\Delta \varphi(x, y)-k^{2} \varphi(x, y)=0
$$

in the sector $-\pi \alpha \leq \theta \leq 0$, satisfying

$$
\frac{\partial \varphi}{\partial y}-\varphi=0 \text { for } \theta=0
$$

${ }^{*}$ Received November 12, 1959. The author wishes to acknowledge support by the Office of Naval Research under Contract Nonr-222(62). 
and

$$
\frac{\partial \varphi}{\partial n}=0 \quad \text { for } \quad \theta=-\pi \alpha
$$

Roseau [11] and Peters [10] have found solutions for this problem for $k<1$ and all angles $\pi \alpha \leq \pi$. Roseau [12] has also studied solutions for angles $\pi \alpha<\pi / 2$ when $k>1$. Some of these solutions, which include the Stokes edge waves, have been considered also by Ursell [15]. The question of determining all solutions of the boundary value problem satisfying appropriate conditions at infinity and at the origin has been studied by Stoker [13] and Weinstein [17] for $0<k<1$ in the case $\alpha=1 / 2$. (See also [14, p. 84].) A proof of uniqueness for $\alpha=1 / 4$ can be constructed by using Roseau's special investigation of that case [11, pp. 27-30] together with Stoker's and Weinstein's methods. In this paper we shall use similar methods to handle the case $\alpha=3 / 4$.

For $k=0$ the waves are two-dimensional. In this case solutions for all angles were found earlier by Isaacson [4]. Stoker [13] has treated the uniqueness question for $\alpha=1 / 2 n$. Brillouët [1] has studied the uniqueness question for $\alpha=p / 2 q$ following the line of Lewy's original investigation [6]. A uniqueness proof for all $\alpha \leq 1$ for $k=0$ can be constructed using the methods and results employed by Lewy in [8].

The present investigation was begun with the aim of finding out the true situation with regard to uniqueness for $k>0$. There is no possibility of answering the question for all angles by the method used here, and even the case of all angles $\pi p / 2 q$, which in principle probably could be handled by the methods employed here, seems uninvitingly complex. One can hope, however, that knowledge of a few special cases may help in guiding a general consideration using methods similar to those employed by Lewy $[7,8,9]$ and the author [5].

2. Reduction to a simpler boundary value problem. We wish to determine for $\alpha=3 / 4$ all solutions of the boundary value problem (1.1)-(1.3) which have continuous second derivatives in the sector $-3 \pi / 4 \leq \theta \leq 0, r>0$ and satisfy two additional boundedness conditions. At infinity we shall require that

$$
|\varphi|+\left|\frac{\partial \varphi}{\partial x}\right|+\left|\frac{\partial \varphi}{\partial y}\right|<M \text { for } r>R_{0},
$$

where $M$ and $R_{0}$ are some positive constants. Near the origin we shall assume that

$$
|\varphi|+\left|\frac{\partial \varphi}{\partial x}\right|+\left|\frac{\partial \varphi}{\partial y}\right|<C r^{-7 / 3+e}
$$

where $C$ and $\epsilon$ are positive constants. Observe that (2.2) allows $\varphi$ to have a logarithmic singularity at the origin but excludes solutions which behave like $r^{-4 / 3}$ at the origin.

Because the normal derivative of $\varphi$ vanishes on $\theta=-3 \pi / 4$, the function $\varphi$ can be extended by reflection to the sector $-3 \pi / 2 \leq \theta \leq 0$. In the larger sector $\varphi$ will again satisfy the conditions (2.1) and (2.2). Similarly, since $\partial \varphi / \partial y-\varphi$ vanishes on $\theta=0$, it can be extended by reflection to the sector $-3 \pi / 4 \leq \theta \leq 3 \pi / 2$. The resulting function $\partial \varphi / \partial y-\varphi$ is multiple-valued when considered as a function of $x$ and $y$, but it can be considered as a single-valued function of $r$ and $\theta$. It satisfies the following two relations uniformly in $\theta$ : 


$$
\begin{array}{lll}
\frac{\partial \varphi}{\partial y}-\varphi=O(1) & \text { for } r \rightarrow \infty, & -\frac{3}{2} \pi \leq \theta \leq \frac{3}{2} \pi \\
\frac{\partial \varphi}{\partial y}-\varphi=O\left(r^{-7 / 3+\epsilon}\right) & \text { for } r \rightarrow 0, & -\frac{3}{2} \pi \leq \theta \leq \frac{3}{2} \pi .
\end{array}
$$

Since the function $\varphi$ is symmetric with respect to the line $\theta=-3 \pi / 4$ and $\partial \varphi / \partial y-$ $\varphi=0$ for $\theta=0$, we conclude that $\partial \varphi / \partial x-\varphi=0$ for $\theta=-3 \pi / 2$. Define

$$
\psi(x, y)=\left(\frac{\partial}{\partial y}-1\right)\left(\frac{\partial}{\partial x}-1\right) \varphi(x, y) .
$$

The function $\psi$ is then a solution of the differential equation $\Delta \psi-k^{2} \psi=0$ for $-3 \pi / 2 \leq$ $\theta \leq 3 \pi / 2$ because the differential operators involved have constant cocfficients. Also $\psi=0$ for $\theta=0$ and for $\theta=-3 \pi / 2$. Thus $\psi$ is a solution of a much simpler boundary value problem.

The method we employ is first to solve this boundary value problem for $\psi$ and then use (2.4) to determine $\varphi$ by the solution of ordinary differential equations. Finally we must verify that the $\varphi$ obtained is a solution of (1.1)-(1.3) and satisfies the boundedness conditions (2.1) and (2.2).

It is possible for all $\alpha=p / 2 q$ with $p$ odd to perform a similar reduction to a problem with vanishing boundary values for a sector with angle $2 \pi \alpha$. This simpler boundary value problem can be solved, (see Brillouët [1]) but since for larger $p$ and $q$ the differential operator used in the reduction is more complicated, the determination of $\varphi$ from the solution of the simpler boundary value problem presents difficulties.

We first use (2.1) and (2.2) together with the following lemma to derive bounds for $\psi$ at the origin and at infinity.

Lemma 1 . Let $R_{1}$ be an arbitrary positive number. Let $u$ be any solution of $\Delta u-k^{2} u=0$ existing in a circle of radius $d<R_{1}$ about $\left(x_{0}, y_{0}\right)$ and let $M_{1}$ be the supremum of $u$ inside this circle. Then

$$
\left|\frac{\partial u}{\partial x}\left(x_{0}, y_{0}\right)\right|+\left|\frac{\partial u}{\partial y}\left(x_{0}, y_{0}\right)\right|<L \frac{M}{d},
$$

where $L$ is a constant dependent only upon the differential equation and the number $R_{1}$.

This lemma is a special case of known theorems. In particular it is a special case of results of Gevrey [3, p. 148] and it also follows from the Schauder interior estimates as stated by Douglis and Nirenberg [2].

We now use the lemma together with (2.3) to obtain estimates for $\psi$ at infinity and at the origin in the sector $-3 \pi / 4 \leq \theta \leq 3 \pi / 4$. For the estimate at infinity we use a circle of radius 1 about the point $(x, y)$. For the estimate at the origin we use a circle of radius $r / 2$ about the point $(x, y)$. We find that the following estimates hold uniformly in $\theta$ :

$$
\begin{array}{lll}
\psi(x, y)=O(1) & \text { for } r \rightarrow \infty, & -\frac{3}{4} \pi \leq \theta \leq \frac{3}{4} \pi, \\
\psi(x, y)=O\left(r^{-10 / 2+\epsilon}\right) & \text { for } r \rightarrow 0, & -\frac{3}{4} \pi \leq \theta \leq \frac{3}{4} \pi .
\end{array}
$$

In the following discussion it will sometimes be convenient to consider $\psi$ as a function of $r$ and $\theta$. To avoid possible confusion we set

$$
\Psi(r, \theta)=\psi(x, y)
$$


Since $\varphi$ is symmetric with respect to the line $\theta=-3 \pi / 4$, the function $\psi$ in view of (2.4) also must be symmetric with respect to $\theta=-3 \pi / 4$. In other words

$$
\Psi(r, \theta)=\Psi\left(r,-\frac{3}{2} \pi-\theta\right) .
$$

By reflection across the line $\theta=0$ we obtain

$$
\Psi(r, \theta)=-\Psi(r,-\theta) .
$$

Combined with (2.6) this yields the information that $\Psi$ is a periodic function of $\theta$ with period $3 \pi$. For any fixed value of $r$ the function $\Psi$ can therefore be expanded in a convergent Fourier series which because of (2.6) and (2.7) must have the form

$$
\Psi(r, \theta)=\sum_{n=0}^{\infty} c_{n}(r) \sin \frac{2}{3}(2 n+1) \theta,
$$

where the coefficients $c_{n}(r)$ are given by

$$
c_{n}(r)=\frac{8}{3 \pi} \int_{-3 \pi / 4}^{0} \Psi(r, \theta) \sin \frac{2}{3}(2 n+1) \theta d \theta .
$$

The function $\Psi(r, \theta)$ satisfies the partial differential equation

$$
\frac{\partial^{2} \Psi}{\partial r^{2}}+\frac{1}{r} \frac{\partial \Psi}{\partial r}+\frac{1}{r^{2}} \frac{\partial^{2} \Psi}{\partial \theta^{2}}-k^{2} \Psi=0 .
$$

Differentiating (2.9) and using this differential equation, we obtain

$$
\begin{aligned}
c_{n}^{\prime \prime}(r)+\frac{1}{r} c_{n}^{\prime}(r)-\left[k^{2}+\left\{\frac{2}{3 r}\right.\right. & \left.(2 n+1)\}^{2}\right] c_{n}(r) \\
& =-\frac{2}{3 \pi r^{2}} \int_{-3 \pi / 4}^{0}\left[\frac{\partial^{2} \Psi}{\partial \theta^{2}}+\left(\frac{2}{3}(2 n+1)\right)^{2} \Psi\right] \sin \frac{2}{3}(2 n+1) \theta d \theta .
\end{aligned}
$$

The right side of this equation is equal to zero, as can be seen by integrating the first term of the integrand by parts twice and using the fact that $\Psi=0$ for $\theta=0$ and $\partial \Psi / \partial \theta=0$ for $\theta=-3 \pi / 4$. It follows that the function $c_{n}(r)$ is a linear combination of modified Bessel functions of order $2(2 n+1) / 3$ that is

$$
c_{n}(r)=A_{n} I_{2(2 n+1) / 3}(k r)+B_{n} K_{2(2 n+1) / 8}(k r),
$$

where $A_{n}$ and $B_{n}$ are real constants.

Next we use the estimates (2.5) to prove that all of these constants except $B_{0}$ and $B_{1}$ are equal to zero. For $\rho \rightarrow \infty$ over positive values the following asymptotic relations hold (see [16, p. 202]):

$$
\begin{aligned}
& I_{\nu}(\rho) \sim(2 \pi \rho)^{-1 / 2} e^{\rho}, \\
& K_{\nu}(\rho) \sim\left(\frac{\pi}{2 \rho}\right)^{1 / 2} e^{-\rho} .
\end{aligned}
$$

Inserting the first estimate of (2.5) into (2.9) and letting $r \rightarrow \infty$, we conclude that $A_{n}=0$ for $n=0,1,2, \cdots$. Also for $\rho \rightarrow 0$ over positive values

$$
K_{,}(\rho)=O\left(\rho^{-\eta}\right),
$$

when $\nu>0[16$, p.77-78]. Hence the second estimate of (2.5) when combined with 
(2.9) allows us to conclude that $B_{n}=0$ for $n=2,3,4, \ldots$. Thus we find

$$
\psi(x, y)=B_{0} K_{2 / 3}(k r) \sin \frac{2}{3} \theta+B_{1} K_{2}(k r) \sin 2 \theta,
$$

where $B_{0}$ and $B_{1}$ are real constants.

3. Solution of ordinary differential equations. In Sec. 2 we proved that if $\varphi(x, y)$ is a solution of the boundary value problem (1.1)-(1.3) satisfying the boundedness conditions (2.1) and (2.2), then the corresponding function $\psi(x, y)$ determined by (2.4) must have the form (2.13). In this section we shall prove that corresponding to each function $\psi$ of the form (2.13) there is at most one function $\varphi$ satisfying (1.1)-(1.3), (2.1) and (2.2), thus establishing that for each $k>0$ there is at most a two-parameter family of solutions of our problem. The method employed in this section is essentially integrating ordinary differential equations.

We shall need the following lemma.

Lemma 2. Suppose $u(x, y)$ is a solution of the differential equation $\Delta u-k^{2} u=0$ in the strip $a<x<b,-\infty<y<\infty$. Suppose also that there is a constant $C_{2}$ such that

$$
|u|+\left|\frac{\partial^{2} u}{\partial x^{2}}\right|+\left|\frac{\partial u}{\partial y}\right|<C_{2}
$$

for $y>y_{0}, a<x<b$. Then the function

$$
U(x, y)=e^{y} \int_{+\infty}^{y} e^{-t} u(x, t) d t
$$

is a solution of the same differential equation in the same strip.

Proof. By differentiating we obtain

$$
\Delta U-k^{2} U=e^{y} \int_{\infty}^{y} e^{-t}\left[\frac{\partial^{2} u(x, t)}{\partial x^{2}}+\left(1-k^{2}\right) u(x, t)\right] d t+u(x, y)+\frac{\partial}{\partial y} u(x, y),
$$

as is easily justified in view of (3.1). Transforming the integrand by using the differential equation and then integrating by parts, we obtain

$$
\begin{aligned}
\Delta U-k^{2} U & =e^{y} \int_{\infty}^{y} e^{-t}\left(u-\frac{\partial^{2} u}{\partial t^{2}}\right) d t+u(x, y)+\frac{\partial}{\partial y} u(x, y) \\
& =e^{y} \int_{\infty}^{y} e^{-t}\left(\frac{\partial u}{\partial t}-\frac{\partial u}{\partial t}\right) d t=\mathbf{0} .
\end{aligned}
$$

The inequality (3.1) insures that the boundary terms at the limit $+\infty$ vanish.

Now let us make a cut along the positive $y$-axis of the $x, y$ plane and restrict $\theta$ to the range $-3 \pi / 2<\theta<\pi / 2$. In this way for given values of $B_{0}$ and $B_{1}$ in (2.13) we obtain a single-valued function $\psi(x, y)$ defined in the entire cut plane. Let

$$
v(x, y)=\left(\frac{\partial}{\partial x}-1\right) \varphi(x, y)
$$

so that $v$ is a solution of the equation

$$
\left(\frac{\partial}{\partial y}-1\right) v(x, y)=\psi(x, y)
$$


Integrating we find

$$
v(x, y)=e^{y} g(x)+e^{y} \int_{\infty}^{\nu} e^{-t} \psi(x, t) d t \equiv e^{y} g(x)+v^{*}(x, y),
$$

where $g$ is a function of $x$ alone. By Lemma $2 v^{*}(x, y)$ is a solution of the differential equation (1.1) for $-\infty<x<0,-\infty<y<\infty$ and also it is a solution for $0<x<\infty$, $-\infty<y<\infty$. As we shall see, $v^{*}(x, y)$ is discontinuous on the entire $y$-axis. Since the function $v$ is also a solution of (1.1), $e^{y} g(x)$ must be a solution, i.e.

$$
g^{\prime \prime}(x)+\left(1-k^{2}\right) g(x)=0
$$

for the intervals $-\infty<x<0$ and $0<x<\infty$. We shall denote by $g_{1}(x)$ and $g_{2}(x)$ the analytic functions of $x$ coinciding with $g$ for $x<0$ and $x>0$, respectively.

By $(2.11) \psi(x, t)$ is exponentially small for $x^{2}+t^{2}$ large. Consequently for $x \rightarrow+\infty$ with $y$ fixed

$$
v(x, y)=e^{y} g_{2}(x)+o(1) .
$$

Also by (3.2) and (2.1) we know $v(x, y)=O(1)$ for $x \rightarrow+\infty, y \leq 0$. When $k<1$, this does not impose any additional condition on $g_{2}(x)$; but if $k>1$ among the solutions of (3.5), $a_{1} e^{k_{1} x}+a_{2} e^{-k_{1} x}$ with $k_{1}=\left(k^{2}-1\right)^{1 / 2}$, we must reject all those for which $a_{1} \neq 0$. Hence we obtain the additional condition

$$
g_{2}^{\prime}(0)=-k_{1} g_{2}(0), \quad k_{1}=\left(k^{2}-1\right)^{1 / 2},
$$

which must be satisfied in order to obtain a $\varphi$ satisfying (2.1). Similarly for $k=1$ we must have $g_{2}^{\prime}(0)=0$. Thus (3.6) must be imposed for $k \geq 1$.

Next, solving (3.2) for $\varphi(x, y)$ in the lower half plane, we obtain

$$
\varphi(x, y)=e^{x} h(y)+e^{x} \int_{\infty}^{x} e^{-t} v(t, y) d t
$$

By an argument using Lemma 2 with $x$ and $y$ interchanged one can show that the second term on the right hand side is a solution of the differential equation (1.1) for $y<0$. It follows that for $y<0$

$$
h^{\prime \prime}(y)+\left(1-k^{2}\right) h(y)=0 .
$$

By (1.2) we must have $\partial \varphi / \partial y-\varphi=0$ for $y=0, x>0$. For all $y<0$ we have

$$
\begin{aligned}
\frac{\partial \varphi}{\partial y}-\varphi & =e^{x}\left(h^{\prime}(y)-h(y)\right)+e^{x} \int_{\infty}^{x} e^{-t}\left(\frac{\partial}{\partial y}-1\right) v(t, y) d t \\
& =e^{x}\left(h^{\prime}(y)-h(y)\right)+e^{x} \int_{\infty}^{x} e^{-t} \psi(t, y) d t .
\end{aligned}
$$

Keeping $x$ fixed $>0$ and taking the limit for $y \rightarrow 0^{-}$, we conclude that we must have

$$
h^{\prime}(0)-h(0)=0 .
$$

Since, as we have seen, $v(t, y)=O(1)$ for $t \rightarrow+\infty$, we obtain from (3.7)

$$
\varphi(x, y)=e^{x} h(y)+O(1)
$$

for $x \rightarrow+\infty$ with $y$ fixed. To have $\varphi$ bounded as required by (2.1) we must have $h^{\prime}(0)=$ $h(0)=0$, and hence by the differential equation $h(y)=0$ for all $y$. 
The boundary condition (1.3) also must be satisfied. For $y=x, x<0$ we have, in view of (3.2) and (3.8),

$$
\begin{aligned}
2^{1 / 2} \frac{\partial \varphi}{\partial n} & =\frac{\partial \varphi}{\partial x}-\frac{\partial \varphi}{\partial y} \\
& =[\varphi(x, y)+v(x, y)]-\left[e^{x} \int_{\infty}^{x} e^{-t} \psi(t, y) d t+\varphi(x, y)\right] \\
& \left.=v(x, y)-e^{x} \int_{\infty}^{x} e^{-t} \psi(y, t) d t \quad \text { (since } \psi(t, y)=\psi(y, t)\right) \\
& =g(x) e^{x}, \text { by }(3.4)
\end{aligned}
$$

Consequently we must have $g_{1}(x)=0$ for all $x$.

Since $v$ and $\partial v / \partial x$ must be continuous across the negative $y$-axis, we must have for $y<0$

$$
\begin{aligned}
& g_{2}(0)=\lim _{x \rightarrow 0^{-}} \int_{\infty}^{\nu} e^{-t} \psi(x, t) d t-\lim _{x \rightarrow 0^{+}} \int_{\infty}^{\nu} e_{n}^{-t} \psi(x, t) d t, \\
& g_{2}^{\prime}(0)=\lim _{x \rightarrow 0^{-}} \int_{\infty}^{\nu} e^{-t} \frac{\partial \psi}{\partial x} d t-\lim _{x \rightarrow 0^{+}} \int_{\infty}^{\nu} e^{-t} \frac{\partial \psi}{\partial x} d t .
\end{aligned}
$$

The function $g_{2}(x)$ is uniquely determined by $g_{2}(0)$ and $g_{2}^{\prime}(0)$ since it is a solution of the differential equation (3.5). In the next section we shall establish that these limits exist for every function $\psi$ of the form (2.13) and are independent of $y$. Without further work, however, we can conclude that if there is a solution corresponding to $\psi$, it is given by the formula

$$
\varphi(x, y)=e^{x+\nu} \int_{\infty}^{x} e^{-t} g(t) d t+e^{x+\nu} \int_{\infty}^{x} \int_{\infty}^{\nu} e^{-t-\tau} \psi(\tau, t) d t d \tau,
$$

where

$$
g(x)=\left\{\begin{array}{lll}
0 & \text { if } & x<0 \\
g_{2}(x) & \text { if } & x>0
\end{array}\right.
$$

Observe that by (3.10) and (3.11) $\varphi$ is uniquely determined by $\psi$ and also that the dependence is linear. From (2.13) we see that for each $k>0$ there are at most two linearly independent solutions of the boundary value problem satisfying the conditions (2.1) and (2.2). In fact, for $k<1$ Roseau [11] and Peters [10] have found two linearly independent solutions, one of which is finite at the origin, and the other of which has a logarithmic singularity there. If one used their work, to complete the proof that for each $\psi$ the formula (3.11) represents a solution of our problem, one would only need to verify that their solutions satisfy the conditions (2.1) and (2.2). However, we shall not do this, but instead we shall give a proof which is independent of their work. At the same time we shall complete the study for $k \geq 1$, where it turns out that (because of (3.6)) there is only one linearly independent solution of the problem and it has a logarithmic singularity at the origin.

To summarize, we have proved that for each $\psi$ of the form (2.13), if the limits (3.10) exist, then the function $\varphi(x, y)$ given by (3.11) is a solution of the boundary value problem (1.1)-(1.3). In the next section we show that the limits (3.10) exist and at the 
same time evaluate them explicitly by using Bessel function formulas. Then in the last section we study the behavior at infinity and at the origin of $\varphi(x, y)$ as given by (3.11) and in doing so verify that it satisfies the conditions (2.1) and (2.2).

4. Evaluation of some limits. We shall use the formula (see [16 p. 388])

$$
\int_{0}^{\infty} e^{-t \cosh \beta} K_{\nu}(t) d t=\frac{\pi}{\sin \nu \pi} \cdot \frac{\sinh \nu \beta}{\sinh \beta}
$$

valid for $0<\nu<1, \operatorname{Re} \cosh \beta>-1,-\pi / 2 \leq \operatorname{Im} \cosh \beta \leq \pi / 2$. It follows that

$$
\int_{0}^{\infty} e^{-t} K_{\nu}(k t) d t=\frac{\pi}{k \sin \nu \pi} \cdot \frac{\sinh \nu \beta}{\sinh \beta}
$$

for $0<\nu<1,0<k<\infty, \cosh \beta=1 / k$ with $0 \leq \operatorname{Im} \cosh \beta \leq \pi / 2$. More precisely, if $k=1$, then $\beta=0$; and the ratio $\sinh \nu \beta / \sinh \beta$ is defined to have the value $\nu$. For $k>1, \beta$ is complex and therefore sometimes it may be more convenient to use the alternative expression

$$
\frac{\pi}{k \sin \nu \pi} \cdot \frac{\sin \nu \gamma}{\sin \gamma}
$$

with $\cos \gamma=1 / k, 0 \leq \gamma<\pi / 2$.

To abbreviate, we introduce a limit operator $\delta$ defined by the equation

$$
\delta f=\lim _{x \rightarrow 0^{+}} f(x, y)-\lim _{x \rightarrow 0^{-}} f(x, y)
$$

for any function $f$ for which the limit exists. Then (3.10) becomes

$$
\begin{aligned}
& g_{2}(0)=\delta \int_{y}^{\infty} e^{-t} \psi(x, t) d t, \\
& g_{2}^{\prime}(0)=\delta \int_{y}^{\infty} e^{-t} \frac{\partial}{\partial x} \psi(x, t) d t
\end{aligned}
$$

provided the limits exist. We shall prove that these limits do exist for every $y<0$ and are independent of $y$. Throughout this section we shall assume that $y<0$.

We investigate separately the contributions due to each term of $\psi$ as given by (2.13). In each case in the following discussion where we obtain a limit of an integral by passing to the limit under the integral sign, this can be justified by the Lebesgue dominated convergence theorem. First we have

$$
\begin{aligned}
& \delta \int_{y}^{\infty} e^{-t} K_{2 / 3}\left(k\left[t^{2}+x^{2}\right]^{1 / 2}\right) \sin \frac{2}{3} \theta d t \\
&=\int_{0}^{\infty} e^{-t} K_{2 / 3}(k t)[\sin (\pi / 3)-\sin (-\pi)] d t=\frac{\pi}{k} \frac{\sinh (2 \beta / 3)}{\sinh \beta} .
\end{aligned}
$$

Using the formula

$$
\frac{\partial^{2}}{\partial x \partial y} K_{0}(k r)=\frac{k^{2}}{2} K_{2}(k r) \sin 2 \theta
$$

and integrating by parts, we find

$$
L_{1} \equiv \delta \int_{y}^{\infty} e^{-t} K_{2}\left(k\left[t^{2}+x^{2}\right]^{1 / 2}\right) \sin 2 \theta d t
$$




$$
\begin{aligned}
& =\delta \int_{y}^{\infty} \frac{2}{k^{2}} e^{-t} \frac{\partial^{2}}{\partial t \partial x} K_{0}\left(k\left[t^{2}+x^{2}\right]^{1 / 2}\right) d t \\
& =\delta \int_{y}^{\infty} \frac{2}{k^{2}} e^{-t} \frac{\partial}{\partial x} K_{0}\left(k\left[t^{2}+x^{2}\right]^{1 / 2}\right) d t .
\end{aligned}
$$

Also (see $[16$, pp. 79,80$]$ )

$$
\frac{\partial}{\partial x} K_{0}(k r)=-\frac{k x}{r} K_{1}(k r)=-\frac{x}{r^{2}}+x p(r),
$$

where there is a constant $C$ such that for $0<r<\infty$

Consequently

$$
|p(r)|<C(1+|\log r|) \text {. }
$$

$$
L_{1}=-\delta \int_{\nu}^{\infty} \frac{2}{k^{2}} e^{-t} \frac{x}{x^{2}+t^{2}} d t+\delta \int_{\nu}^{\infty} \frac{2}{k^{2}} e^{-t} x p\left(\left[t^{2}+x^{2}\right]^{1 / 2}\right) d t .
$$

The second term is 0 since the limit of the integrand is 0 for $x \rightarrow 0$. The first term can be evaluated by introducing $\tau=t / x$ as a new variable of integration. Since $y<0$, we obtain

$$
\begin{aligned}
L_{1} & =-\delta \int_{y}^{\infty} \frac{2}{k^{2}} e^{-t} \frac{x}{x^{2}+t^{2}} d t=-\frac{4}{k^{2}} \lim _{x \rightarrow 0^{+}} \int_{y / x}^{\infty} \frac{e^{-\tau x}}{1+\tau^{2}} d \tau \\
& =-\frac{4}{k^{2}} \int_{-\infty}^{\infty} \frac{d \tau}{1+\tau^{2}}=-\frac{4 \pi}{k^{2}} .
\end{aligned}
$$

From the formula (see $[16$, p. 79])

$$
z K_{p}^{\prime}(z)+\nu K_{\nu}(z)=-z K_{\nu-1}(z)
$$

one can derive the following identity:

$$
\frac{\partial}{\partial x}\left(K_{\nu}(k r) \sin \nu \theta\right)=\frac{\partial}{\partial y}\left(K_{\nu}(k r) \cos \nu \theta\right)-k K_{,-1}(k r) \sin (\nu-1) \theta .
$$

Using this identity and the fact that $K_{-v}(z)=K_{\nu}(z)$, we obtain

$$
\begin{aligned}
\delta \int_{y}^{\infty} e^{-t} \frac{\partial}{\partial x}\left\{K_{2 / 3}\left(k\left[t^{2}+x^{2}\right]^{1 / 2}\right)\right. & \left.\sin \frac{2}{3} \theta\right\} d t \\
= & \delta \int_{\nu}^{\infty} e^{-t} \frac{\partial}{\partial t}\left\{K_{2 / 3}\left(k\left[t^{2}+x^{2}\right]^{1 / 2}\right) \cos \frac{2}{3} \theta\right\} d t \\
& +\delta \int_{\nu}^{\infty} k e^{-t} K_{1 / 3}\left(k\left[t^{2}+x^{2}\right]^{1 / 2}\right) \sin \frac{1}{3} \theta d t \equiv L_{2}+L_{3} .
\end{aligned}
$$

The limit $L_{2}$ can be evaluated by first integrating by parts and then passing to the limit under the integral sign to obtain by (4.1)

Also

$$
L_{2}=\int_{0}^{\infty} e^{-t} K_{2 / 3}(k t)[\cos (\pi / 3)-\cos (-\pi)] d t=\frac{3^{1 / 2} \pi}{k} \frac{\sinh \frac{2}{3} \beta}{\sinh \beta} .
$$

$$
L_{3}=k \int_{0}^{\infty} e^{-t} K_{1 / 3}\left(k \sqrt{t^{2}+x^{2}}\right)[\sin (\pi / 6)-\sin (-\pi / 2)] d t=3^{1 / 2} \pi \frac{\sinh (\beta / 3)}{\sinh \beta} .
$$


We have

$$
\begin{aligned}
L_{4} \equiv & \delta \int_{\nu}^{\infty} e^{-t} \frac{\partial}{\partial x}\left(K_{2}\left(k\left[t^{2}+x^{2}\right]^{1 / 2}\right) \sin 2 \theta\right) d t \\
& =\delta \int_{\nu}^{\infty} \frac{2 e^{-t}}{k^{2}} \frac{\partial^{3}}{\partial t \partial x^{2}}\left(K_{0}\left(k\left[t^{2}+x^{2}\right]^{1 / 2}\right)\right) d t \\
& =\delta \int_{\nu}^{\infty} \frac{2}{k^{2}} e^{-t} \frac{\partial^{2}}{\partial x^{2}} K_{0}\left(k\left[t^{2}+x^{2}\right]^{1 / 2}\right) d t .
\end{aligned}
$$

Since $K_{0}(k r)$ is a solution of $\Delta K_{0}-k^{2} K_{0}=0$, we obtain

$$
\begin{aligned}
L_{4} & =\delta \int_{\nu}^{\infty} 2 e^{-t} K_{0}\left(k\left[t^{2}+x^{2}\right]^{1 / 2}\right) d t-\delta \int_{\nu}^{\infty} \frac{2 e^{-t}}{k^{2}} \frac{\partial^{2}}{\partial t^{2}} K_{0}\left(k\left[t^{2}+x^{2}\right]^{1 / 2}\right) d t \\
& =\delta \int_{\nu}^{\infty}\left(2-\frac{2}{k^{2}}\right) e^{-t} K_{0}\left(k\left[t^{2}+x^{2}\right]^{1 / 2}\right) d t=0,
\end{aligned}
$$

since the limit of the integrand is the same for $x \rightarrow 0^{+}$as it is for $x \rightarrow 0^{-}$.

Thus

$$
\begin{aligned}
& g_{2}(0)=B_{0} \frac{\pi}{k} \frac{\sinh (2 \beta / 3)}{\sinh \beta}-B_{1} \frac{4 \pi}{k^{2}} \\
& g_{2}^{\prime}(0)=B_{0}\left\{\frac{3^{1 / 2} \pi}{k} \frac{\sinh (2 \beta / 3)}{\sinh \beta}+3^{1 / 2} \pi \frac{\sinh (\beta / 3)}{\sinh \beta}\right\}
\end{aligned}
$$

where $\cosh \beta=1 / k, \operatorname{Re} \beta \geq 0,0 \leq \operatorname{Im} \beta \leq \pi / 2$. It is easily verified that the coefficients of $B_{0}$ in these expressions never vanish.

When combined with (3.6) these formulas establish that for $k \geq 1, B_{0}$ is determined uniquely if $B_{1}$ is given. For example for $k=1$ we must have $B_{0}=0$. Thus for $k \geq 1$ there is at most one linearly independent solution satisfying the conditions (2.1) and (2.2).

5. Behavior at infinity and at the origin. We now investigate the behavior at infinity and at the origin of $\varphi(x, y)$ as given by (3.11), (2.13), (4.3), and (3.5). We begin by estimating

$$
v^{*}(x, y)=e^{y} \int_{\infty}^{y} e^{-t} \psi(x, t) d t
$$

(see (3.4)). If $\psi$ is any one of the functions given by (2.13) and $p$ is an arbitrary positive number, then there is by (2.11) a corresponding number $c_{1}$ such that

$$
|\psi(x, y)|<c_{1} e^{-k r} \text { for } r=\left(x^{2}+y^{2}\right)^{1 / 2} \geq p .
$$

(It is necessary to omit a neighborhood of the origin because of the singularity of $K$, there). For $y \geq 0, x^{2}+y^{2} \geq p^{2}$ we obtain

$$
\left|v^{*}(x, y)\right| \leq e^{\nu} \int_{\nu}^{\infty} c_{1} e^{-(k+1) t} d t \leq \frac{c_{1}}{k+1} e^{-k y} .
$$

For $y<0, x^{2}+y^{2} \geq p^{2}, x \neq 0$ we write

$$
v^{*}(x, y)=e^{y} \int_{\infty}^{-D} e^{-t} \psi(x, t) d t+e^{y} \int_{-p}^{y} e^{-t} \psi(x, t) d t \equiv T_{1}+T_{2} .
$$


By the results of the last section we know the integral in $T_{1}$ approaches a limit as $x \rightarrow 0^{+}$ and also approaches a limit as $x \rightarrow 0^{-}$. Furthermore by (5.2) the integral in $T_{1}$ approaches 0 as $|x| \rightarrow \infty$. Hence there is a number $c_{2}$ such that $\left|T_{1}\right|<c_{2} e^{\nu}$.

For the second term $T_{2}$ we have by (5.2)

$$
\left|T_{2}\right|<c_{1} e^{\nu}\left|\int_{-p}^{\nu} e^{(k-1) t} d t\right|
$$

which is less than or equal to

$$
\frac{C_{1} e^{y}}{|k-1|}\left\{e^{(k-1) \nu}+e^{-(k-1) \nu}\right\}
$$

for $k \neq 1$ and is less than or equal to

$$
c_{1} e^{\nu}(|y|+p)
$$

for $k=1$. In either case, if we define

$$
\mu= \begin{cases}k & \text { for } \quad k<1 \\ 1-\eta & \text { for } \quad k=1 \\ 1 & \text { for } \quad k>1\end{cases}
$$

with $\eta$ an arbitrarily small positive number, then there is a constant $c_{3}$ independent of $x$ and $y$ such that $\left|T_{2}\right|<c_{3} e^{\mu \nu}$. Combining this estimate with that for $\left|T_{1}\right|$ and (5.3) we find

$$
\left|v^{*}(x, y)\right|<c_{4} e^{-\mu|y|} \text { for } x \neq 0, \quad x^{2}+y^{2} \geq p^{2} .
$$

Since for $y \leq 0$,

$$
\varphi(x, y)=e^{x+y} \int_{\infty}^{x} e^{-t} g(t) d t+e^{x} \int_{\infty}^{x} e^{-t} v^{*}(t, y) d t,
$$

we obtain immediately

$$
\left|\varphi(x, y)-e^{x+y} \int_{\infty}^{x} e^{-t} g(t) d t\right|<c_{4} e^{\mu \nu} .
$$

For $k<1$ with $k_{2}=\left(1-k^{2}\right)^{1 / 2}$ we have

$$
g_{2}(t)=g_{2}(0) \cos k_{2} t+k_{2}^{-1} g_{2}^{\prime}(0) \sin k_{2} t,
$$

and hence for $x \geq 0$

$$
\begin{aligned}
e^{x} \int_{\infty}^{x} e^{-t} g(t) d t=\frac{1}{\left(2-k^{2}\right)}\left\{-\left(g_{2}(0)+g_{2}^{\prime}(0)\right) \cos k_{2} x\right. \\
\left.+\left(k_{2}^{2} g_{2}(0)-g_{2}^{\prime}(0)\right) k_{2}^{-1} \sin k_{2} x\right\},
\end{aligned}
$$

while for $x<0$ since $g(t)=0$ for $t<0$,

$$
e^{x} \int_{\infty}^{x} e^{-t} g(t) d t=e^{x} \int_{\infty}^{0} e^{-t} g_{2}(t) d t=\frac{-e^{x}}{\left(2-k^{2}\right)}\left(g_{2}(0)+g_{2}^{\prime}(0)\right) .
$$

If $k \geq 1$, assuming (3.6) is satisfied, we have

$$
g_{2}(t)=g_{2}(0) e^{-k_{1} t},
$$


where $k_{1}=\left(k^{2}-1\right)^{1 / 2}$. Hence for $x \geq 0$

$$
e^{x} \int_{\infty}^{x} e^{-t} g(t) d t=\frac{-g_{2}(0) e^{-k_{1} x}}{1+k_{1}}
$$

while for $x<0$

$$
e^{x} \int_{\infty}^{x} e^{-t} g(t) d t=\frac{-g_{2}(0) e^{x}}{1+k_{1}} .
$$

Thus we see that for $y \leq 0, \varphi(x, y)$ is bounded outside a neighborhood of the origin for every function $\psi$ given by (2.13) in case $k<1$; and the same is true for $k \geq 1$ provided (3.6) holds.

To study the behavior on the free surface $y=0$ it is desirable to use a better estimate for $v^{*}$ there. By (5.2) for $x>p$ we have $|\psi(x, t)|<c_{1} e^{-k x}$. It follows that $\left|v^{*}(x, 0)\right|<$ $c_{1} e^{-k x}$ and hence for $y=0$ the right side of (5.6) can be replaced by $c_{1} e^{-k x} /(k+1)$. Equation (5.7) then shows that for $k<1, \varphi(x, 0)$ approaches a sinusoidal wave as $x \rightarrow+\infty$. Similarly for $k=1, \varphi(x, 0)$ approaches a constant as $x \rightarrow+\infty$, the constant being $-g_{2}(0)$. For $k>1, \varphi(x, 0)=O\left(e^{-k_{1} x}\right)$ as $x \rightarrow+\infty$.

To complete the verification that condition (2.1) is satisfied we must verify that $|\partial \varphi / \partial x|$ and $|\partial \varphi / \partial y|$ also are bounded. By (3.2), (3.4), and (3.8) we have

$$
\begin{aligned}
& \frac{\partial \varphi}{\partial x}=\varphi(x, y)+v(x, y)=\varphi(x, y)+e^{y} g(x)+v^{*}(x, y), \\
& \frac{\partial \varphi}{\partial y}=\varphi(x, y)+e^{x} \int_{\infty}^{x} e^{-t} \psi(t, y) d t=\varphi(x, y)+v^{*}(y, x)
\end{aligned}
$$

since $\psi(y, t)=\psi(t, y)$. The boundedness then follows in view of the estimate (5.5) for $v^{*}$.

Next we study the behavior of $\varphi$ near the origin. In view of (2.13), it is convenient to consider separately two different functions $\psi$,

$$
\begin{aligned}
& \psi_{0}(x, y)=K_{2 / 3}(k r) \sin (2 \theta / 3), \\
& \psi_{1}(x, y)=\frac{k^{2}}{2} K_{2}(k r) \sin 2 \theta=\frac{\partial^{2}}{\partial x \partial y} K_{0}(k r),
\end{aligned}
$$

and obtain estimates for the corresponding functions $v_{0}^{*}$ and $v_{1}^{*}$.

We have

$$
v_{0}^{*}(x, y)=e^{\nu} \int_{\infty}^{\nu} e^{-t} K_{2 / 3}\left(k\left[x^{2}+t^{2}\right]^{1 / 2}\right) \sin (2 \theta / 3) d t=O(1)
$$

for all $x$ and all $y \leq 0$.

On the other hand,

$$
\begin{aligned}
v_{1}^{*}(x, y) & =e^{y} \int_{\infty}^{\nu} e^{-t} \frac{\partial^{2}}{\partial t \partial x} K_{0}\left(k\left[t^{2}+x^{2}\right]^{1 / 2}\right) d t \\
& =\frac{\partial}{\partial x} K_{0}\left(k\left[x^{2}+y^{2}\right]^{1 / 2}\right)+e^{y} \int_{\infty}^{\nu} e^{-t} \frac{\partial}{\partial x} K_{0}\left(k\left[t^{2}+x^{2}\right]^{1 / 2}\right) d t \\
& =\frac{\partial}{\partial x} K_{0}(k r)+O(1)
\end{aligned}
$$


for $x^{2}+y^{2} \rightarrow 0, y \leq 0$, as can be seen by the argument used to evaluate $L_{1}$ in the previous section. Hence

$$
\begin{aligned}
e^{x} \int_{\infty}^{x} e^{-t} v^{*}(t, y) d t & =B_{1} e^{x} \int_{\infty}^{x} e^{-t} \frac{\partial}{\partial t} K_{0}\left(k\left[t^{2}+y^{2}\right]^{1 / 2}\right) d t+O(1) \\
& =K_{0}(k r)+e^{x} \int_{\infty}^{x} e^{-t} K_{0}\left(k\left[t^{2}+y^{2}\right]^{1 / 2}\right) d t+O(1) \\
& =K_{0}(k r)+O(1)
\end{aligned}
$$

for $x^{2}+y^{2} \rightarrow 0$. Hence we obtain

$$
\varphi(x, y)=\frac{2}{k^{2}} B_{1} K_{0}(k r)+O(1)
$$

for $r \rightarrow 0$. Thus if $B_{1}=0$ we obtain a solution which is finite at the origin; if $B_{1} \neq 0$ the solution will have a logarithmic singularity at the origin.

To complete the verification of the condition (2.2) one uses (5.11) to estimate the derivatives.

In summary, for $k<1$ there are two linearly independent solutions satisfying the conditions (2.1) and (2.2). One can be taken to be finite at the origin. The other then has a logarithmic singularity there. At infinity for $y=0$ both of these solutions have a sinusoidal form. On the other hand for $k \geq 1$, there is only one linearly independent solution satisfying the conditions (2.1) and (2.2) and it has a logarithmic singularity at the origin. For $k=1$ with $y=0$ this solution approaches a constant for $x \rightarrow+\infty$; for $k>1$ the solution dies out exponentially as $x \rightarrow \infty$. In every case the solutions die out exponentially as $y \rightarrow-\infty$.

\section{BIBLIOGRAPHY}

1. G. Brillouët, Etude de quelques problemes sur les ondes liquides de gravite, Publ. Scientif. Tech., Ministère de l'Air, Paris, No. 329 (1957)

2. A. Douglis and L. Nirenberg, Interior estimates for elliptic systems of partial differential equations, Communs. Pure Appl. Math. 8, 503-538 (1955)

3. M. Gevrey, Sur la nature analytique des solutions des équations aux dérivées partielles, Ann. École Norm. 35, 129-190 (1918)

4. E. Isaacson, Water waves over a sloping bottom, Communs. Pure Appl. Math. 3, 11-31 (1950)

5. R. S. Lehman, Developments in the neighborhood of the beach of surface waves over an inclined bottom, Communs. Pure Appl. Math. 7, 393-439 (1954)

6. H. Lewy, Water waves on sloping beaches, Bull. Am. Math. Soc. 52, 737-775 (1946)

7. H. Lewy, Developments at the confluence of analytic boundary conditions, Univ. of California Publications in Mathematics, 1, 247-280 (1950)

8. H. Lewy, The development of functions associated with surface waves over an inclined bottom, Tech. Rept. No. 26, ONR Contract Nonr-225(11), Appl. Math. and Statist. Lab., Stanford University, 1954

9. H. Lewy, On linear difference-differential equations with constant coefficients, J. Math. and Mech. 6, 91-108 (1957)

10. A. S. Peters, Water waves over sloping beaches and the solution of a mixed boundary value problem for $\Delta \varphi-k^{2} \varphi=0$ in a sector, Communs. Pure Appl. Math. 5, 87-108 (1952)

11. M. Roseau, Contribution d la theorie des ondes liquides de gravite en profondeur variable, Publ. Scientif. Tech., Ministère de l'Air, Paris, No. 275 (1952) 
12. M. Roseau, Short waves parallel to the shore over a sloping beach, Communs. Pure Appl. Math. 11, 433-493 (1958)

13. J. J. Stoker, Surface waves in water of variable depth, Quart. Appl. Math. 5, 1-54 (1947)

14. J. J. Stoker, Water waves, Interscience Publishers Inc., New York, 1957

15. F. Ursell, Edge waves on a sloping beach, Proc. Roy. Soc. A214, 79-97 (1952)

16. G. N. Watson, A treatise on the theory of Bessel functions, 2nd ed., Cambridge University Press, 1944

17. A. Weinstein, On surface waves, Can. J. Math. 1, 271-278 (1949) 